hep-ph/9906421

UMD-PP-99-118

\title{
Searching for Strongly Interacting Massive Particles (SIMPs)
}

\author{
R. N. Mohapatra ${ }^{1}$, F. Olness ${ }^{2}$, R. Stroynowski², V. L. Teplitz ${ }^{2}$ \\ ${ }^{1}$ Department of Physics, University of Maryland, College Park, MD, 20742 \\ ${ }^{2}$ Department of Physics, Southern Methodist University, Dallas, TX 75275
}

(May, 1999)

\begin{abstract}
We consider laboratory experiments that can detect stable, neutral strongly interacting massive particles (SIMPs). We explore the SIMP annihilation cross section from its minimum value (restricted by cosmological bounds) to the barn range, and vary the mass values from a $\mathrm{GeV}$ to a $\mathrm{TeV}$. We calculate, as a function of the SIMP-nucleon cross section, the minimum nucleon number $A$ for which there should be binding in a nucleus. We consider accelerator mass spectrometry with a gold $(A=200)$ target, and compute the likely abundance of anomalous gold nuclei if stable neutral SIMPs exist. We also consider the prospects and problems of detecting such particles at the Tevatron. We estimate optimistically that such detection might be possible for SIMPs with SIMP-nucleon cross sections larger than 0.1 millibarn and masses between 25 and $50 \mathrm{GeV}$.
\end{abstract}

\section{INTRODUCTION}

The nature of the dark matter in the universe is a question of great interest for particle physics since the successful standard model has no candidate with the right properties to qualify as one. It is, therefore, hoped that determining the properties of the dark matter particle can provide important information about the nature of new physics beyond the standard model. One intriguing candidate that has been discussed in literature is a stable massive strongly interacting neutral particle (SIMP) [1]. Such particles arise in many gauge models [2], and their existence is of interest even if they do not constitute dark matter. While there already exist several severe constraints from cosmology and astrophysics on these particles, [1, 目 they can still be viable if their strong interaction parameters are appropriately chosen. It is therefore of interest to explore all possible avenues to "tighten the noose" on them. In this paper we discuss two experiments of interest in this connection.

The essential property of the SIMPs (henceforth denoted as $X$ ) that determines their possible experimental signature is the nature of their interaction with ordinary nucleons. It is not easy to guess the interactions of such unknown particles; however, we have some guide from nucleon-hyperon interactions. In addition, as detailed in Section II, we make use of 
an approximate relation between $\sigma_{X N}$ and $\sigma_{X \bar{X}}$. Furthermore, cosmology provides a lower bound on $\sigma_{X \bar{X}}$. In considering binding of $X$ in nuclei, we will assume the XN potential to be attractive at low energies, as should be the case whenever $X N$ scattering is a result of two particle exchange. [6]

In a recent publication [1] two of us (RNM and VLT) investigated constraints on SIMPs from searches for anomalous nuclei containing them. We considered two cases. First we built on the work of Dicus and Teplitz, [5] assuming that the depth of binding potential $V_{X N}$ is of the order of a few MeV's, as is the case for the $\Lambda$ particles. That work showed that the anomalous nucleus ${ }^{9} B e_{X}^{*}$ would be formed during big bang nucleosynthesis (BBN) if the $X-N$ potential is attractive and large enough (i.e., $10 \mathrm{MeV}$ or more) for $\mathrm{X}$-binding in light nuclei. Using a plausible model for the $X \bar{X}$ annihilation cross section, Ref. [4] estimated that the concentration of the $X$ particles relative to the baryons must be bigger than $\sim 10^{-5}$, which then leads to a concentration of ${ }^{9} B e_{X}^{*}$ in the present universe which is several orders of magnitude higher than the present upper limits on anomalous $B e$ obtained in experiments by Hemmick et al. [7]

Second, Reference [4] pointed out that, for a given attractive $X-N$ potential, binding is more likely in the more massive high $\mathrm{Z}$ nuclei than in low $\mathrm{Z}$ nuclei. This is because, by the uncertainty principle, there is less kinetic energy for the SIMP in the high Z nucleus. We noted, however, the absence of data for anomalous nuclei with $\mathrm{Z}$ above that of sodium $(\mathrm{A}=23$, $\mathrm{Z}=11$ ), and urged that experiments be undertaken in high $\mathrm{Z}$ nuclei. Since publication of Ref. [4], we have been informed [8] that one group hopes to look for anomalous gold $(A=200$, $\mathrm{Z}=79$ ) nuclei using accelerator mass spectrometry.

The purpose of this paper is to build upon Ref [4] in two directions. First we compute $X$ binding and abundance in gold as a function of $M_{X}$ and $\sigma_{X N}$. We choose gold in view of the experiment of Ref. [8]. Results for other heavy nuclei will be similar. Second, we make an initial estimate for detection of $X-\bar{X}$ pairs at the Fermilab Tevatron. The plan of the paper is as follows: in Section II we estimate the abundance of anomalous gold as a function of $M_{X}$ and $\sigma_{X N}$ for the region of the $\left\{M_{X}, \sigma_{X N}\right\}$ plane for which there is binding. In Section III we consider Tevatron detection. Section IV gives our conclusions.

\section{SEARCHING IN GOLD}

We perform two calculations in this section. First, we compute $X-A$ binding as a function of nucleon number $A$ and $M_{X}$ for a range of $\sigma_{X N}$. Second, we compute the abundance of anomalous gold, relative to normal gold, as a function of $M_{X}$ and $\sigma_{X N}$.

We consider first the nuclei in which the X-particle will bind. As in Ref. [四] and works cited therein, we take the $\mathrm{X}-\mathrm{A}$ binding energy to be:

$$
B_{X}=V_{X N}-\frac{\pi^{2}}{2 \mu R^{2}}
$$

where $V_{X N}$ is the average $\mathrm{X}-\mathrm{N}$ potential at rest, $\mu$ is the reduced mass of the $\mathrm{X}$-A system, and $\mathrm{R}$ is the size of the nucleus in which $X$ is bound. We have used a simple particle in a box model. We take $R \simeq R_{0} A^{1 / 3}$ with $R_{0} \simeq 1.4$ fermi, and $A$ is the atomic number of the nucleus. The reduced mass of the $\mathrm{X}-\mathrm{A}$ system is given by the usual expression: 


$$
\frac{1}{\mu}=\frac{1}{M_{A}}+\frac{1}{M_{X}}
$$

The average $\mathrm{X}-\mathrm{N}$ potential, $V_{X N}$, is defined implicitly via:

$$
\frac{V_{X N}}{V_{N N}}=\sqrt{\frac{\sigma_{X N}}{\sigma_{N N}}}
$$

If we take the low energy NN scattering cross section $\sigma_{N N}$ to be on the order of 1 barn, use $V_{N N} \simeq 50 \mathrm{MeV}$, and assume $B_{X} \ll V_{X}$, we can write equation (11) as

$$
\frac{2 R_{0}^{2}}{\pi^{2}} V_{N N} \sqrt{\frac{\sigma_{X N}}{\sigma_{N N}}} A^{2 / 3}=\frac{1}{M_{A}}+\frac{1}{M_{X}}
$$

which numerically reduces to

$$
\frac{1}{2 G e V} \sqrt{\frac{\sigma_{X N}}{1 \text { barn }}} A^{2 / 3}=\frac{1}{M_{A}}+\frac{1}{M_{X}}
$$

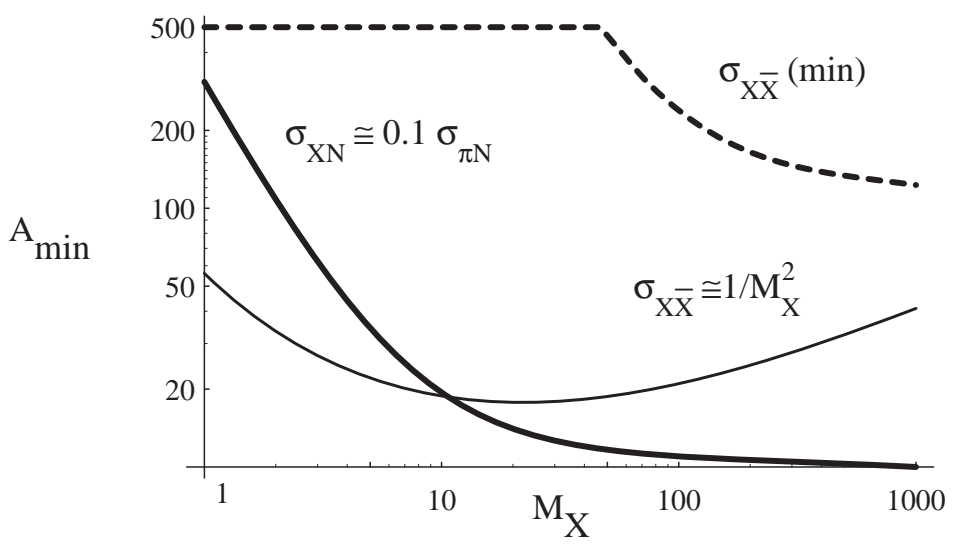

FIG. 1. The results of solving equation (5) for three choices for $\sigma_{X N}$. The dotted line is for $\sigma_{X \bar{X}}(\min )$, the thin line is for $\sigma_{X \bar{X}} \simeq 1 / M_{X}^{2}$, and the thick line is for $\sigma_{X N} \simeq \sigma_{\pi N}$.

In Figure 1, we give the results of solving equation (5) for three choices for $\sigma_{X N}$. We determine the minimum value of $\mathrm{A}$ for which there is binding as a function of $M_{X}$. We assume an approximate factorization hypothesis of ref. [4],

$$
\beta \sigma_{X \bar{X}} \sigma_{N N} \simeq \sigma_{X N}^{2}
$$

where $\beta \sim 1$.

To be definite, we shall consider three values for $\sigma_{X \bar{X}}$.

1. We consider $\sigma_{X \bar{X}} \sim 3 \times 10^{-13}$ barn as a lower bound. This is the minimum cross section allowed which does not overclose the universe, (assuming $M_{X} \gtrsim \mathrm{GeV}$ ).

2. We use the estimate of ref. [3], $\sigma_{X \bar{X}} \simeq 1 / M_{X}^{2}=0.4 m b / M_{X}^{2}(G e V)$ 
3. Finally we look at the estimate of ref. [9], $\sigma_{X N} \simeq 0.1 \sigma_{\pi N} \sim 2 \mathrm{mb}$. This estimate takes $X$ to be the particle responsible for ultra-high energy cosmic ray (UHECR) events [10], the UHECRON.

For case (1), the minimum $\sigma_{X \bar{X}}$, we see in Figure 1 that gold will only capture X's for $M_{X} \gtrsim 80$, (assuming the interaction is attractive). For case (2), $\sigma_{X \bar{X}} \sim 1 / M_{X}^{2}$, we estimate there is always binding in relatively light nuclei, In particular, much of the $M_{X}$ range $(15 \sim 150$ $\mathrm{GeV}$, roughly) would have binding in sodium, and hence is likely ruled out by current experiments. For case (3), the UHECRON, there is binding in gold for $M_{X}$ above a few $\mathrm{GeV}$.

We turn now to the less straightforward problem of estimating the abundance of anomalous gold. Our first requirement is a capture scenario. Two possibilities present themselves. First, the gold on Earth was likely made by a supernova in the giant molecular cloud (GMC) in which the sun was bound. GMC's live for tens of million of years, so one could assume capture in the GMC before formation with an exposure time on the order of $10^{7}$ years. There are complications, however. The $X$ needs to be slowed from the galactic virial velocity down to thermal velocities, and then captured. In addition, both the capture reaction, $X+A \rightarrow{ }^{X} A+\gamma$, and the dissociation reaction, $\gamma+{ }^{X} A \rightarrow X+A$ from $\gamma^{\prime}$ s generated by hot stars and supernova in the GMC, must be considered. There appear to be too many unknown parameters for a reliable estimate. The second, more conservative and more easily estimated scenario is to assume SIMP capture after formation of the Earth.

The key to the second scenario is that gold nuclei be close enough to the surface for galactic halo SIMPs to reach them. Geologists say, with some confidence, [1] that there are selected gold deposits that should have had long exposure to any cosmic SIMPs. These are deposits found at the surface of the earth, in particular, in the gravel of streams ("placer gold") in regions that are sufficiently inactive geologically that one can be reasonably confident of an exposure for over $\sim 10^{7}$ years. The Sierra Nevada Mountains are such a region. Placer gold from the California gold rush would have a lesser exposure time, on the order of $\sim 10^{4}$ years. For such placer gold, the results discussed below on the abundance of anomalous gold should be decreased by a factor of $10^{3}$.

Given an exposure time of $\sim 10^{7}$ years, we must then calculate how deep into the Earth the $X$ particle penetrates, and how many nuclei are in the region penetrated. We make the conservative approximation that the gold is uniformly mixed with other elements so $X$ can be captured by any $A>A_{\text {min }}$. We assume all X's are captured since scattering slows them to thermal velocities.

\begin{tabular}{||c|c|c|c||}
\hline \hline Range of $A$ & $A_{M}$ & Element & $\mathrm{C}_{\mathrm{M}}$ in $(\mu \mathrm{gm} / \mathrm{gm})$ \\
\hline \hline$\leq 20$ & 20 & $A r$ & $5 \times 10^{5}$ \\
\hline $10 \leq A \leq 26$ & 26 & $A l$ & $8.3 \times 10^{4}$ \\
\hline $26 \leq A \leq 137$ & 137 & $B a$ & 425 \\
\hline $137 \leq A \leq 200$ & 207 & $P b$ & 12.5 \\
\hline \hline
\end{tabular}

TABLE I. Abundances of the most abundant nuclei in a given A-interval. 
We now compute the fraction $f$ of gold that has captured an $\mathrm{X}$ :

$$
f\left(M_{X}, \sigma_{X N}\right) \simeq n_{X} v t \frac{f_{A u}}{N_{A u}}
$$

Here $n_{X}$ is the galactic halo abundance at the solar distance from the galactic center, $v$ is the galactic virial velocity $(300 \mathrm{~km}), t$ is the exposure time, $f_{A u}$ is the fraction of the stopped X's captured in gold, and $N_{A u}$ is the number of Au nuclei $\left(\right.$ per $\left.\mathrm{cm}^{2}\right)$ in the stopping length $\lambda_{X}$ of $\mathrm{X}$.

The fraction $f_{A u}$ is taken to be:

$$
f_{A u}=\frac{C_{A u}}{C_{M}} \sqrt[3]{\frac{A_{M}}{A_{A u}}}
$$

where the $C$ 's are concentrations by weight in the Earth's crust, and we expect the capture cross to be proportional to $A^{2 / 3}$. The index $\mathrm{M}$ designates the most abundant element with a nucleus heavier than $A_{\text {min }}$. We choose $A_{M}$ from Table I.

$N_{A u}$ in equation (7), the number of Au nuclei $\left(\right.$ per $\left.\mathrm{cm}^{2}\right)$ in the stopping length $\lambda_{X}$ of $\mathrm{X}$, is given by

$$
N_{A u}=\lambda_{X} \frac{M_{E}}{m_{p}} \frac{C_{A u}}{A_{A u}}
$$

where $M_{E}$ is the mass of the earth, and $m_{p} \sim 1 \mathrm{GeV}$ is the mass of an ordinary nucleon.

For $\lambda_{X}$ we take

$$
\lambda_{X}=\left\{\frac{\rho_{E}}{\widetilde{A} m_{p}} \widetilde{A}^{2 / 3} \sigma_{X N}\left[1-\left(\frac{M_{X}}{M_{X}+\widetilde{A} m_{p}}\right)^{2}\right]\right\}^{-1}
$$

where $\widetilde{A} \sim 20$ is the $A$ value for an "average nucleus," and the last factor $\left(\equiv \Delta E_{X} / E_{X}\right)$ assumes that $X A$ scattering in the C.M. is isotropic. Note, as a sanity check, that the $C_{A u}$ factor in equation(9) cancels with that in $f_{A u}$, equation(8), as one would expect.

Finally, to get $n_{X}$ in equation(8) we compute the primordial X freeze-out abundance, $\tilde{n}_{X}$, precisely as specified in Kolb and Turner [12], using (in their notation) $\mathrm{n}=1$ and $\mathrm{g}=1$, and making use of the factorization approximation relating $\sigma_{X \bar{X}}$ to $\sigma_{N X}$. We then assume that X's are concentrated in the galaxy to the same extent as baryons, but are distributed in an isothermal spherical halo of $70 \mathrm{kpc}$ radius. This gives $n_{X} / n_{B}=3.7 \times 10^{-3} \tilde{n}_{X} / \tilde{n}_{B}$ with, the tilde denoting the cosmic average.

The result of the calculation for $\log _{10}(1 / f)$ is shown with quiet drama in Table 2. $\sigma_{X N}$ ranges from $5 \times 10^{-7} b$ (corresponding to $\sigma_{X \bar{X}}=\sigma_{\min }=3 \times 10^{-37} \mathrm{~cm}^{2}$ ) to 1 barn, while $M_{X}$ ranges from $1 \mathrm{GeV}$ to $1 \mathrm{TeV}$. In the first column we see that the $X$ does not bind in gold until $M_{X} \in[80,130] \mathrm{GeV}$, which is consistent with Figure 1. As $\sigma_{X N}$ increases, the abundance falls because [12] $Y_{\infty} \propto 1 /\left(M_{X} \sigma_{X \bar{X}}\right)$ and $\sigma_{X \bar{X}} \sim \sigma_{N X}^{2}$. This fact also plays a major role in the decrease of $f$ as $M_{X}$ increases. Note from the table that the boundary between binding and no-binding can be approximated by the implicit relation $M_{X}^{2} \sigma_{X N} \sim 5 m b \mathrm{GeV}^{2}$.

The result of Table 2 is optimistic in that previous experiments have reached abundance fractions approaching $f \sim 10^{-20}$. Therefore, it is likely to be possible to explore the entirety 
of the parameter space of Table 2 in which there is binding of the X. For the UHECR case of Ref. [9] $\left(\sigma_{X N} \gtrsim 0.1 \sigma_{\pi N}, M_{X} \lesssim 50 \mathrm{GeV}\right)$, it is only necessary to go to $10^{-16}$, not $10^{-20}$. This is because Table 2 shows that, even for the largest XN cross sections, if the value of $M_{X}$ is below $50 \mathrm{GeV}, \log _{10}(f)>-15.7$.

While Table 2 shows the fraction $f$ is a relatively smoothly varying function of $\sigma_{N X}$ and $M_{X}$ over most of the range, closer examination shows some regions of large variation. For example, the first column shows a change by 2 orders of magnitude between $M_{X}=350$ and $570 \mathrm{GeV}$. In Figure 1 this corresponds to $M_{X} \simeq 530$ where $A_{\min }$ goes from above 137 to below 137. This corresponds to $A_{M}$ in equation(5) falling from $207(\mathrm{~Pb})$ to $137(\mathrm{Ba})$ which shifts $C_{M}$ in equation(8) by a factor of 35 . To see this effect more clearly, we plot in Figure 2 abundance fraction $f$ as a function of $M_{X}$ for the 3 cross section choices of Figure 1 . The curve for $\sigma_{X \bar{X}}(\min )$ has a steep rise just past $M_{X}=100$ where $A_{M}$ in Figure 2 first falls below 200 (so that there is binding in gold). The discontinuous behavior at $M_{X}=530 \mathrm{GeV}$ is seen clearly. The curve for $\sigma_{X N} \simeq \sigma_{\pi N}$ is monotonic (after its initial rise when binding occurs) corresponding to the monotonic behavior of $\mathrm{Au}$ in Figure 1 for the corresponding curve. The curve for $\sigma_{X \bar{X}} \simeq 1 / M_{X}^{2}$ has discontinuities of both signs corresponding to the fall of this same curve in Figure 1 to below 26 and 20, and subsequent rise above these points.

\begin{tabular}{||c||c|c|c|c|c|c|c|c|c|c|c|c|c|c|c||}
\hline \hline & 0.0005 & 0.0015 & 0.0042 & 0.012 & 0.032 & 0.09 & 0.25 & 0.69 & 1.9 & 5.3 & 15 & 41 & 110 & 310 & 860 \\
\hline \hline 1.0 & - & - & - & - & - & - & - & - & - & 6.3 & 8.3 & 8.7 & 12.5 & 12.9 & 13.4 \\
\hline 1.6 & - & - & - & - & - & - & - & - & 6.1 & 8.1 & 8.5 & 12.3 & 12.7 & 13.1 & 13.6 \\
\hline 2.7 & - & - & - & - & - & - & - & 5.9 & 7.9 & 8.3 & 12.1 & 12.5 & 12.9 & 13.3 & 13.8 \\
\hline 4.3 & - & - & - & - & - & - & 5.7 & 7.7 & 8.1 & 11.1 & 12.3 & 12.7 & 13.1 & 13.6 & 14.0 \\
\hline 7.1 & - & - & - & - & - & 5.5 & 7.5 & 7.9 & 10.9 & 12.1 & 12.5 & 12.9 & 13.4 & 13.8 & 14.2 \\
\hline 12 & - & - & - & - & 5.6 & 7.6 & 8.1 & 8.5 & 12.2 & 12.7 & 13.1 & 13.5 & 13.9 & 14.3 & 14.8 \\
\hline 19 & - & - & - & - & 7.5 & 7.9 & 8.3 & 11.3 & 12.5 & 12.9 & 13.3 & 13.8 & 14.2 & 14.6 & 15.0 \\
\hline 31 & - & - & - & 7.4 & 7.8 & 8.2 & 8.6 & 12.4 & 12.8 & 13.2 & 13.6 & 14.1 & 14.5 & 14.9 & 15.3 \\
\hline 50 & - & - & 5.7 & 7.7 & 8.1 & 8.5 & 11.5 & 12.7 & 13.1 & 13.6 & 14.0 & 14.4 & 14.8 & 15.3 & 15.7 \\
\hline 81 & - & 5.7 & 7.7 & 8.1 & 8.5 & 8.9 & 11.9 & 13.1 & 13.5 & 14.0 & 14.4 & 14.8 & 15.2 & 15.6 & 16.1 \\
\hline 132 & 5.7 & 7.7 & 8.1 & 8.5 & 8.9 & 9.3 & 12.2 & 13.5 & 13.9 & 14.3 & 14.7 & 15.2 & 15.6 & 16.0 & 16.4 \\
\hline 220 & 6.0 & 8.0 & 8.4 & 8.9 & 9.3 & 9.7 & 12.6 & 13.9 & 14.3 & 14.7 & 15.1 & 15.5 & 16.0 & 16.4 & 16.8 \\
\hline 350 & 6.4 & 8.4 & 8.8 & 9.3 & 9.7 & 10.1 & 13.8 & 14.3 & 14.7 & 15.1 & 15.5 & 15.9 & 16.4 & 16.8 & 17.2 \\
\hline 570 & 8.4 & 8.8 & 9.2 & 9.7 & 10.1 & 10.5 & 14.3 & 14.7 & 15.1 & 15.5 & 15.9 & 16.4 & 16.8 & 17.2 & 17.6 \\
\hline 930 & 8.9 & 9.3 & 9.7 & 10.1 & 10.5 & 10.9 & 14.7 & 15.1 & 15.5 & 16.0 & 16.4 & 16.8 & 17.2 & 17.6 & 18.1 \\
\hline \hline
\end{tabular}

TABLE II. $M_{X}$ (vertical) is in units of $\mathrm{GeV}$, and $\sigma_{X N}$ (horizontal) is in units of $m b$. Table entries are $\log _{10}(1 / f)$, and the - indicates those cases for which $X$ does not bind at all. 


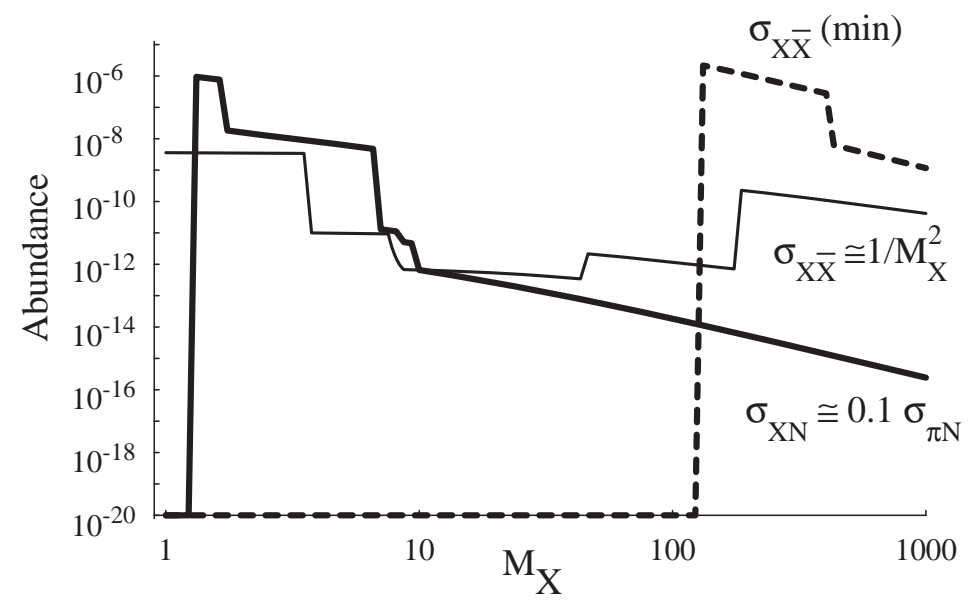

FIG. 2. The abundance fraction $f$ as a function of $M_{X}$ for the 3 cross section choices. The dotted line is for $\sigma_{X \bar{X}}(\min )$, the thin line is for $\sigma_{X \bar{X}} \simeq 1 / M_{X}^{2}$, and the thick line is for $\sigma_{X N} \simeq \sigma_{\pi N}$.

It is also of interest to make a connection with the experiments searching for weakly interacting massive particles (WIMPs). For $\sigma_{X N} \gtrsim 4 \times 10^{-6} b$, which corresponds to $\sigma_{X \bar{X}}$ a factor of 50 above $\sigma_{X \bar{X}}(\mathrm{~min})$, the contribution of $X$ to the local galactic dark matter density $\left(4 \times 10^{-25} \mathrm{~g} / \mathrm{cm}^{3}\right)$ is less than 2 percent.

It is important to note that we only assume that the mass density of SIMPs is less than or equal to the dark matter constraint; we do not assume, as did Starkman et al., [1], that it saturates the constraint.

In summary, we see from the above discussion that: i) we have binding in gold for $M_{X}^{2} \sigma_{X N} \gtrsim 5 m b \mathrm{GeV}^{2}$; ii) a SIMP of mass up to a TeV satisfying this condition, and having an attractive interaction with nucleons, should form anomalous gold nuclei of sufficient abundance to be seen by accelerator mass spectrometer experiments sensitive to one part in $10^{20}$; and iii) UHECRs could be detected in an experiment with a sensitivity of one part in $10^{16}$ for the mass range from a few $\mathrm{GeV}$ to their $50 \mathrm{GeV}$ maximum, (assuming a target of gold with $10^{7}$ years' exposure).

\section{SEARCHING AT THE TEVATRON}

If neutral, stable SIMPs were actually to exist, it might be possible to produce and detect them at the Tevatron. Although such detection might be difficult, further study is required before one can decide whether it is impossible. In this section, we make an optimistic estimate of the most promising ranges of $M_{X}$ and $\sigma_{X N}$ for observing these SIMPs. While the region accessible to the Tevatron is quite restricted, it includes a portion of a particularly interesting region - the region relevant to the explanation of the Ultra High Energy Cosmic Ray (UHECRs) events proposed by Farrar, Kolb and coworkers. [9]

We consider $X \bar{X}$ production in one of the Tevatron detectors. Assuming that the SIMP has colored constituents (e.g., gluon, gluino, ...), its pair production will be accompanied by soft hadrons. A neutral, stable SIMP particle will not generate a signal in the central tracker, and is unlikely to interact in the electromagnetic calorimeter of the Tevatron detectors. It may, however, interact with material in the denser hadronic calorimeter. Such an interaction 
will have kinematical characteristics that can be used to distinguish it on a statistical basis from neutron and $K_{L}^{0}$ interactions. The distinction is based on the assumption that at the Tevatron energies, the mass of a SIMP represents a substantial fraction of its total energy. We then need to ask how much energy the SIMP is likely to deposit in the hadron calorimeter, whether we can distinguish a SIMP shower from that from a neutron or $K_{L}^{0}$, and what overall event rate might be expected.

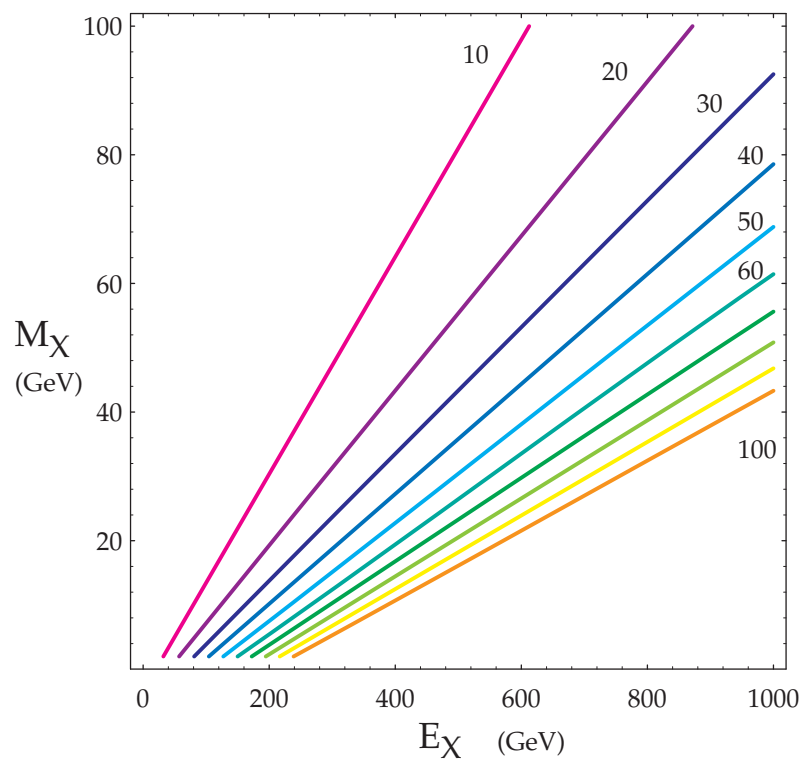

FIG. 3. Contours for energy loss as a function of $\left\{M_{X}, E_{X}\right\}$. The contours displayed are in steps of $10 \mathrm{GeV}$.

With regard to energy deposition in the hadron calorimeter, we make a qualitative estimate. Assume that, in interacting with a nucleon in one of the steel plates, the $X$ loses half of its C.M. kinetic energy into particle production. We can then transform back to the lab system and estimate the energy lost by $X$ as a function of $E_{X}$ and $M_{X}$. Figure 3 shows the results. It has contours for energy losses (into hadron showers) of $10,20, \ldots 100$ $\mathrm{GeV}$. We see that $400 \mathrm{GeV}$ partons could, triggering on multiple $\sim 10 \mathrm{GeV}$ showers, permit discovery of SIMPs with masses up to $60 \mathrm{GeV}$. Similarly $600 \mathrm{GeV}$ partons could push the SIMP discovery limit up to $100 \mathrm{GeV}$. At $300 \mathrm{GeV}$, we can get up to $50 \mathrm{GeV}$ masses, which is the upper limit, in the analysis of Farrar et al [9], of a SIMP that would explain the UHECR events.

If $\sigma_{X N}$ is too small, there will be no showers at all. In $1 m$ of steel, we get about 5 interactions for a cross section of a few $m b$, but only 0.001 for our minimum $\sigma_{X N}(\sim$ $5 \times 10^{-31} \mathrm{~cm}^{2}$ ). We might detect one jet from the SIMP pair at a sufficient rate if we had a cross section on the order of at least a tenth of a millibarn, and both jets for a cross section over a millibarn. The estimate of reference [9] states that a cross section of more than a millibarn is needed if the SIMP is to reproduce the UHECR events.

We estimate the production rate of SIMPs by scaling the production rate of jets by the ratio of $X N$ to the Meson- $\mathrm{N}$ cross sections. We observe that the total cross section for $\pi p$ and $K p$ scattering is approximately $\sim 30 \mathrm{mb}$ for $\sqrt{s}$ of a few hundred $\mathrm{GeV}$, with a mild (logarithmic) variation with $\sqrt{s}$. With this perspective, we can now turn from the $t$-channel 
$X p \rightarrow X p$ process to the $s$-channel $p \bar{p} \rightarrow X \bar{X}$ to estimate a scaled cross section. In the low energy region, $\mathrm{X}$-production will be suppressed relative to jet production by the $X$ mass. However, for $E_{X}$ larger than a few times $M_{X}$, there is no more phase space suppression, and we therefore expect scaling to work well (for $M_{X}=50 \mathrm{GeV}$ ) in the region $E_{X} \approx 200 \mathrm{GeV}$. The cross section for producing jets with $E_{T} \gtrsim 200 \mathrm{GeV}$ is $\sim 100 \mathrm{pb}$. This gives $\sim 3 p b$ for any one quark (to be compared with a t-quark production cross section of $\sim 5 p b$ ), corresponding to $\sim 300$ events in Run I and $\sim 6000$ events in Run II. Again, reference [9] estimates that the UHECR would need a cross section of about a tenth the meson nucleon cross section. Scaling the production cross section by that amount gives a good number of events in Run II.

Turning to the question of whether we would recognize a SIMP shower in a hadron calorimeter if we saw one, the best available discriminant would appear to be the opening angle of the shower. A pion moving transverse in the SIMP-nucleon C.M. system will have a laboratory angle of

$$
\tan \theta=\frac{1}{\gamma}=\frac{\sqrt{2 m_{p} E_{X}+M_{X}^{2}}}{E_{X}+m_{p}}
$$

Comparing a $500 \mathrm{GeV}$ SIMP with a $500 \mathrm{GeV}$ neutron, we see that the SIMP shower will be $\sim 40$ percent wider if $M_{X} \gtrsim 25 \mathrm{GeV}$.

In summary, an optimistic scenario finds the Tevatron discovery potential in the range $\sigma_{X N} \gtrsim 0.1 \mathrm{mb}$ and $25 \mathrm{GeV} \lesssim M_{X} \lesssim 50 \mathrm{GeV}$. Consequently, it might be possible to resolve the UHECR puzzle at Fermilab.

\section{CONCLUSIONS}

We emphasize here that, as discussed in Ref. [⿴囗⿰丿㇄口], searches for SIMPs in anomalous nuclei are much better carried out in high $\mathrm{Z}$ nuclei. In such nuclei, the SIMP kinetic energy is minimized so that the chances of binding are maximized. Table 2 shows that a proposed accelerator mass spectrometry experiment in gold is capable of discovering or eliminating SIMPs over $\sim 80$ percent of the relevant portion of the $\left(\sigma_{X N}, M_{X}\right)$ plane so long as the low energy X-N potential is attractive, (the likely event). [9]

Looking for $X$ at the Tevatron is difficult. One needs events in which there are no high energy particles at the vertex or in the electromagnetic calorimeter, but there are showers in the hadron calorimeter. The range of $M_{X}$ values for which the hadron calorimeter showers are sufficiently energetic goes up to 50 or $100 \mathrm{GeV}$ for the $M_{X}$ mass. The range for which the shower opening angles are sufficiently wide to distinguish them from neutron or Kaon showers begins around $25 \mathrm{GeV}$ for the $M_{X}$ mass. Although this region is limited, it includes at least half the region of interest for a possible UHECR explanation. [9]

Acknowledgments: We thank D. Berley, K. Brockett, K. De, D. Dicus, M.A. Doncheski, R. Ellsworth, G. Farrar, E. T. Herrin, D. Rosenbaum, R. Scalise, and G. Yodh. The work of RNM has been supported by the National Science Foundation grant under no. PHY9802551. The work of Olness, Stroynowski, and Teplitz is supported by DOE. 


\section{REFERENCES}

[1] G. Starkman, A. Gould, R. Esmilzadeh and S. Dimopoulos, Phys. Rev. D 41, 3594 (1990); E. Nardi and E. Roulet, Phys. Lett. B245, 105 (1990); S. Chivukula et al. Phys. Rev. Lett. 65, 957 (1990); R. N. Mohapatra and S. Nussinov, Phys. Rev. D 57, 1940 (1997).

[2] R. N. Mohapatra and S. Nandi, Phys. Rev. Lett. 79, 181 (1997); Z. Chacko, B. Dutta, R. N. Mohapatra and S. Nandi, Phys. Rev. D 56, 5466 (1997); S. Raby, Phys. Rev. D 56, 2852 (1997); S. Barr, D. Chang and G. Senjanović, Phys. Rev. Lett. 67, 2765 (1991); Z. Chacko, M. Luty, E. Ponton and I. Maksymyk, hep-ph/9905390; For tests of such models in colliders, see C. S. Li, P. Nadolsky, C. P. Yuan and H. Y. Zhou, Phys.Rev. D58, 095004,(1999); H. Baer, K. Cheung and J. Gunion, Phys.Rev. D59, 075002, (1999);

[3] R. N. Mohapatra and S. Nussinov, Phys. Rev. D 57, 1940 (1997).

[4] R. N. Mohapatra and V. L. Teplitz, Phys. Rev. Lett. 81, 3079 (1998).

[5] D. A. Dicus and V. L. Teplitz, Phys. Rev. Lett. 44, 218 (1990).

[6] We appreciate a conversation with Professor Farrar in which she emphasized the fact that two particle exchange dominance makes XN scattering attractive rather generally.

[7] T. Hemmick, et al., Phys. Rev. D 41, 2074 (1990).

[8] E. Fishbach, private communication.

[9] D. Chung, G. Farrar and E. W. Kolb, Phys.Rev. D57, 4606, (1998); I.F.M. Albuquerque , G. Farrar, and E. W. Kolb, Phys.Rev. D59, 015021 (1999).

[10] For recent reviews, see N. Hayashida et al. Proc. of International symposium on Extremely High Energy Cosmic Rays, Tokyo, Japan, September (1996); V. Berezinsky, Nucl. Phys. Proc. Suppl. 70, 419 (1999).

[11] E.T. Herrin, private communication.

[12] E. W. Kolb and M. Turner, The Early Universe, Addison-Wesley, Menlo Park, Ca. (1990). 